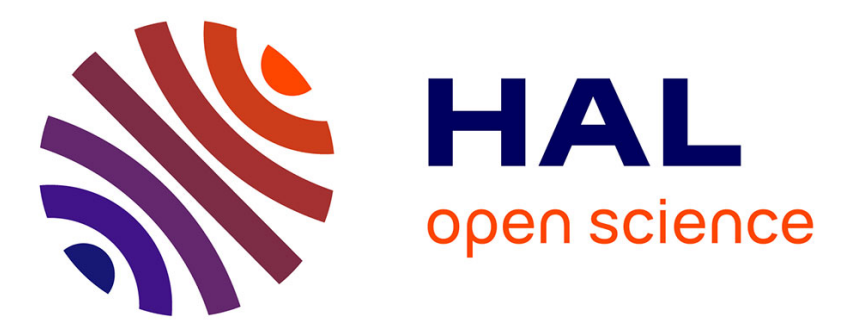

\title{
Two-photon transitions in triazole based quadrupolar and octupolar chromophores: a TD-DFT investigation
}

Claudine Katan, Sergei Tretiak, Jacky Even

\section{To cite this version:}

Claudine Katan, Sergei Tretiak, Jacky Even. Two-photon transitions in triazole based quadrupolar and octupolar chromophores: a TD-DFT investigation. SPIE Photonics Europe 2010, Apr 2010, Bruxelles, Belgium. pp.77123D, 10.1117/12.862574 . hal-00516696

\section{HAL Id: hal-00516696 \\ https://hal.science/hal-00516696}

Submitted on 10 Sep 2010

HAL is a multi-disciplinary open access archive for the deposit and dissemination of scientific research documents, whether they are published or not. The documents may come from teaching and research institutions in France or abroad, or from public or private research centers.
L'archive ouverte pluridisciplinaire HAL, est destinée au dépôt et à la diffusion de documents scientifiques de niveau recherche, publiés ou non, émanant des établissements d'enseignement et de recherche français ou étrangers, des laboratoires publics ou privés. 


\title{
Two-photon transitions in triazole based quadrupolar and octupolar chromophores: a TD-DFT investigation.
}

\author{
Claudine Katan*a,b, Sergei Tretiak ${ }^{\mathrm{c}}$, and Jacky Even ${ }^{\mathrm{b}}$ \\ ${ }^{a}$ CNRS, Université de Rennes 1, Chimie et Photonique Moléculaires (CPM), 35042 Rennes, France \\ ${ }^{\mathrm{b}} \mathrm{CNRS}$, INSA de Rennes, Fonctions Optiques pour les Technologies de l'informatiON (FOTON), \\ CS70839, 35708 Rennes, France \\ ${ }^{c}$ Center for NonLinear Studies (CNLS), and Center for Integrated NanoTechnologies (CINT), Los \\ Alamos, NM USA 87545
}

\begin{abstract}
Simultaneous absorption of two photons has gained increasing attention over recent years as it opens the way for improved and novel technological capabilities. In the search for adequate materials that combine large two-photon absorption (TPA) responses and attributes suitable for specific applications, the multibranch strategy has proved to be efficient. Such molecular engineering effort, based on the gathering of several molecular units, has benefited from various theoretical approaches. Among those, the Frenkel exciton model has been shown to often provide a valuable qualitative tool to connect the optical properties of a multibranched chromophore to those of its monomeric counterpart. In addition, recent extensions of time-dependent density functional theory (TD-DFT) based on hybrid functionals have shown excellent performance for the determination of nonlinear optical (NLO) responses of conjugated organic chromophores and various substituted branched structures. In this paper, we use these theoretical approaches to investigate the one- and two-photon properties of triazole-based chromophores. In fact, experimental data were shown to reveal quite different behaviors as compared to related quadrupolar and octupolar compounds. Our theoretical findings allow elucidating these differences and contribute to the general understanding of structure-property relations. This work opens new perspectives towards synergic TPA architectures.
\end{abstract}

Keywords: two-photon absorption, TPA, NLO, time dependent density functional theory, TD-DFT, Frenkel exciton model, triazole, multibranched chromophores

\section{INTRODUCTION}

The search for versatile nonlinear optical (NLO) chromophores with tunable and intense two-photon absorption (TPA) spectra has motivated huge synthetic, experimental and theoretical efforts over the last decade. ${ }^{1-4}$ The route known as the branching strategy, based on the gathering of either dipolar ${ }^{4-10}$ or quadrupolar ${ }^{4,11-16}$ molecular units via a common conjugated core has proved to be efficient. This molecular engineering effort has benefited from various theoretical approaches through their ability to rationalize structure-property relationships. ${ }^{8-12,16-25}$ Among those, the Frenkel exciton model has been shown to often provide a valuable qualitative tool to connect the photophysical properties of branched chromophores to those of their monomeric counterpart. ${ }^{8-10,17-19}$ However, subtle effects such as noticeable dissymmetry introduced by the central connecting moiety can effectively render the Frenkel exciton model unusable. ${ }^{16}$ In such cases, but also in the quest for quantitative description, accurate prediction and thorough analysis, adiabatic time-dependent density functional theory (TD-DFT) is currently the method of choice for calculating the excited state structure of large molecular systems. ${ }^{4,25-30}$ Recently, branching and "click" chemistry strategies have been combined to design a series of TPA active chromophores built from triazole moieties. ${ }^{31,32}$ Obtained quadrupolar and three-branched chromophores have shown to combine high solubility, full transparency, long excited state lifetimes and strong TPA optical responses in the visible region particularly suitable for optical limiting in the visible region. ${ }^{31,32}$ Experimental data reveal quite different behaviors, both for linear and nonlinear responses, as compared to related quadrupolar or octupolar compounds or between the triazole-based quadrupole and its three-branched analog. The aim of this paper is to investigate theoretically these triazole-based chromophores ${ }^{31}$ and their dipolar units ${ }^{32}$ to get a better understanding of the effect of insertion of a triazole ring on structural, linear and NLO properties as well as within the branching strategy. Theoretical approaches rely on the Frenkel exciton model and TD-DFT calculations using the density matrix formalism for NLO responses. ${ }^{24}$ To illustrate the nature of underlying excited states involved in one and two-photon absorption, we refer to Natural Transition Orbital (NTO) analysis. ${ }^{33}$ 


\section{THEORETICAL APPROACHES}

\subsection{Frenkel exciton model}

To connect the photophysical properties of multibranched chromophores to those of their single-branch counterpart, the Frenkel exciton model has been proposed and adopted, ${ }^{4,17-19}$ offering a reduced description compared to "supramolecular" approaches. It describes the limit of tightly bound localized excitons, as opposed to the Wannier limit of diffuse delocalized excitons. It assumes that all intermolecular interactions between monomers are electrostatic, and short range interactions such as direct wavefunction overlaps are disregarded. Thus, the wavefunction basis for the multibranched system is the direct product of the local wavefunctions relevant for each molecular unit. The reduced Frenkel exciton Hamiltonian is given by:

$$
H=\sum_{i} \hbar \omega_{i} b_{i}^{\dagger} b_{i}+\sum_{i \neq j} V_{i j}\left(b_{i}^{\dagger} b_{j}+b_{j} b_{i}^{\dagger}\right) \text {. }
$$

Indices $i$ and $j$ run over the molecular units, $b_{i}^{\dagger}\left(b_{i}\right)$ are creation (annihilation) operators of a local excitation on the $i$-th site, $\hbar \omega_{i}$ is the transition energy on the $i$-th site, and $V_{i j}$ is the matrix element describing coupling between sites $i$ and $j$. Because the electrostatic interaction decreases quickly with the distance between monomers, often only the nearest neighbor contribution is retained. For a multibranched system made of identical molecular units, all hopping integrals become equal, $V_{i j}=V$. In the case of dipolar branches, the approximation of two-level monomer is usually reasonable. Interbranch interaction results in a splitting of the initially degenerate single excitations. For a homo-dimeric chromophore (e.g. quadrupole), the one-exciton transitions are separated by $2 V$ (Figure 1). Within $C_{i}$ symmetry, the lowest excited state is one-photon allowed and two-photon forbidden and vice versa for the second excited state. For a homo-trimeric chromophore with $C_{3}$ symmetry (e.g. octupole), a two-fold degenerate first excited state, mainly onephoton allowed, is obtained and is shifted by $-V$ from the monomer excited state. The third excited state shifted by $+2 V$ is one-photon forbidden and two-photon allowed (Figure 1).
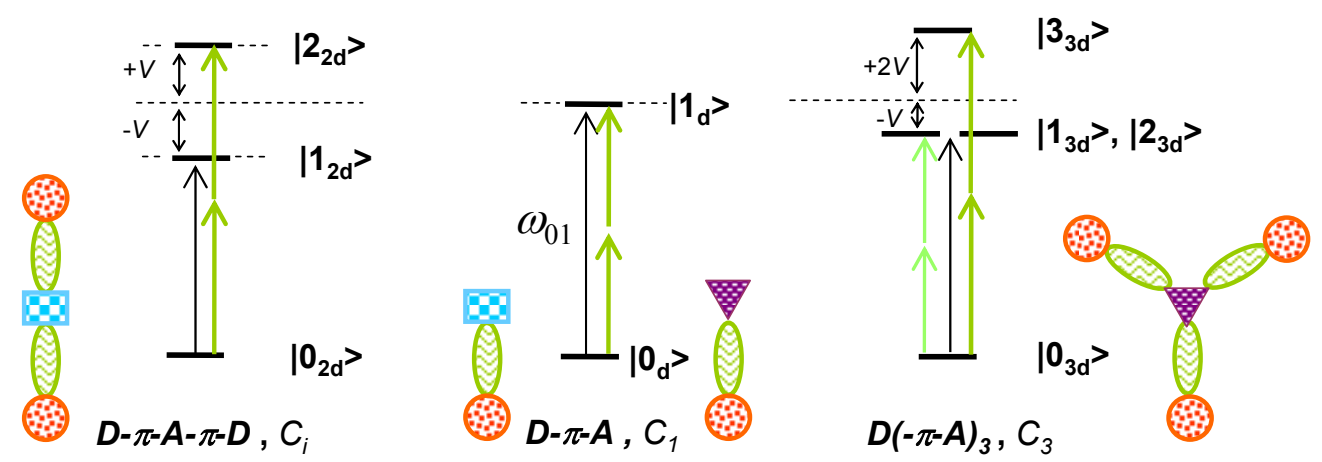

Figure 1. Schematic electronic level diagrams within the excitonic model for quadrupole (left), dipolar molecular unit (middle) and octupole (right). $\left|0_{j}\right\rangle$ denotes the ground state and $\left|n_{j}\right\rangle$ the $n^{\text {th }}$ excited state where $j=\mathrm{d}, 2 \mathrm{~d}, 3 \mathrm{~d}$ respectively for dipolar, quadrupolar, octupolar structures. $V$ denotes the interbranch coupling for the first monomeric excited state.

In general, the Frenkel exciton model provides a reasonable qualitative picture when applied to branched structures. However, when applied to homologous series of dipoles, octupoles and multi-branched systems, quantitative agreement with experimental observations such as enhancement of non linear responses often fails. ${ }^{4,8,9,17}$ In addition, this model has also been shown to be unusable due to the appearance of new excited states resulting from inherent breaking of symmetry of the quadrupolar unit in a three-branched quadrupolar chromophore. ${ }^{16}$ 


\subsection{Computational details of Time Dependent Density Functional Theory (TD-DFT) calculations}

$$
\left(\begin{array}{lll}
\mathrm{Hex}_{2} \mathrm{~N} & \mathbf{d}_{1}: n=1, \mathrm{R}=\mathrm{C}_{6} \mathrm{H}_{5} & \mathbf{2} \mathbf{d}_{\mathbf{1}}: n=2, \mathrm{R}=- \\
& \mathbf{d}_{\mathbf{2}}: n=1, \mathrm{R}=\mathrm{N}\left(\mathrm{C}_{6} \mathrm{H}_{5}\right)_{2} & \mathbf{3 \mathbf { d } _ { 2 }}: n=3, \mathrm{R}=1,3,5-\mathrm{C}_{6} \mathrm{H}_{3}
\end{array}\right.
$$

Scheme 1. Molecular structures of dipolar $\mathbf{d}_{\mathbf{1}}$ and $\mathbf{d}_{\mathbf{2}}$, quadrupolar $\mathbf{2} \mathbf{d}_{\mathbf{1}}$, and three-branched $\mathbf{3} \mathbf{d}_{\mathbf{2}}$ chromophores.

The four chromophores investigated in this work are dipole $\mathbf{d}_{1}\left(\mathrm{C}_{22} \mathrm{H}_{20} \mathrm{~N}_{4}\right)$ and quadrupole $2 \mathbf{d}_{1}\left(\mathrm{C}_{32} \mathrm{H}_{30} \mathrm{~N}_{8}\right)$ shown in Figure 2, dipole $\mathbf{d}_{\mathbf{2}}\left(\mathrm{C}_{28} \mathrm{H}_{25} \mathrm{~N}_{5}\right)$ and three-branched chromophore $\mathbf{3} \mathbf{d}_{\mathbf{2}}\left(\mathrm{C}_{48} \mathrm{H}_{45} \mathrm{~N}_{13}\right)$ shown scheme 1. For the sake of simplicity, the hexyl solubilizing chains of original chromophores ${ }^{31,32}$ have been replaced by methyl groups. Solvation effects were neglected. ${ }^{4}$ Ground-state optimized geometries have been obtained using the Gaussian $98^{34}$ or Gaussian $03^{35}$ packages. For ground state geometries, we previously found that the Hartree-Fock (HF) method is superior to the DFTbased approaches by reproducing accurately bond length alternation parameter in similar conjugated systems when compared to experiment. ${ }^{25}$ Thus, all ground-state geometries are obtained at the HF level using the 6-31G basis set. We note that using DFT-based kernels (such as B3LYP) for ground-state geometry optimizations would lead to consistent red-shifts in the excitation energies and slightly increased one photon absorption (OPA) and TPA response amplitudes compared to respective quantities, calculated at HF level.

Hybrid functionals such as B3LYP have been found to be very accurate for excited states in many molecular systems. ${ }^{28,29,36}$ Consequently, we used TD-B3LYP/6-31G level of theory to investigate linear and NLO properties using the density matrix formalism for NLO responses as described in ref. ${ }^{24}$ This method has already demonstrated its relevance $^{4,16,37}$ for both linear and nonlinear absorption spectra in different series of substituted chromophores. Excitedstate electronic structures were calculated with Gaussian $03,{ }^{35}$ including 20 excited states for dipoles, 30 for the quadrupolar compound and 40 for the three-branched chromophore, The number of excited states was found to be sufficient as asymptotic values are reached for the absorption spectra and only small effects (e.g. 10\% for the first band using 24 excited states for $\mathbf{3 d}_{\mathbf{2}}$ ) are observed on the amplitude of the TPA spectra. Vertical transition frequencies, dipoles and densities are used to model both OPA and TPA spectra. ${ }^{24}$ In particular, the third order polarizability $\gamma(-\omega$; $\omega$; $\omega ;-\omega)$ has been calculated using the density-matrix formalism as described in ref. ${ }^{24}$ The TPA cross-section $\sigma_{2}(\omega)$ in vacuum is related to the imaginary part of the orientational average of $\gamma(-\omega ; \omega ; \omega ;-\omega):^{4}$

$$
\begin{aligned}
& \langle\gamma\rangle=\frac{1}{15}\left(3 \sum_{i} \gamma_{i i i i}+\sum_{j \neq i}\left(\gamma_{i i j j}+\gamma_{i j i j}+\gamma_{i j j i}\right)\right), \\
& \sigma_{2}(\omega)=\frac{24 \pi^{2} \hbar \omega^{2}}{c^{2}} \operatorname{Im}\langle\gamma\rangle,
\end{aligned}
$$

where $\hbar$ is Planck's constant and $c$ is the speed of light. The damping factor $\Gamma$ introduced in $\gamma_{\mathrm{ijkl}}$ to simulate finite linewidths in the resonant spectra has been fixed to $0.20 \mathrm{eV}$ for all chromophores. It is important to notice that all comparisons with the experimental response amplitudes are subject to uncertainties related to the choice of the linebroadening parameter and to consideration of solvation effects. ${ }^{4}$ Natural transition orbital (NTO) analysis of the excited states $^{33}$ has been used to analyze the nature of the excited states involved in the photophysical processes. They offer the most compact representation of a given transition density in terms of an expansion into single-particle transitions. Figures showing molecular geometries and NTOs were obtained with XcrySDen. ${ }^{38}$

\section{RESULTS AND DISCUSSION}

\subsection{Molecular geometry and ground state properties}

The branching effect of triazole based compounds has been studied with model chromophores $\mathbf{2} \mathbf{d}_{\mathbf{1}}\left(\mathrm{Figure}_{2}\right)$ and $\mathbf{3} \mathbf{d}_{\mathbf{2}}$ (Figure 3 ) that correspond to the branching of respectively two dipoles $\mathbf{d}_{\mathbf{1}}$ (Figure 2) via the biphenyl (BP) moiety and three dipoles $\mathbf{d}_{\mathbf{2}}$ (Figure 3) through the triphenylamine (TPamine) branching center. The ground state optimal geometries of the branched chromophores show geometrical parameters for each branch quasi-identical to those of their 
dipolar counterpart. Among the different possible conformations of quadrupole $\mathbf{2} \mathbf{d}_{1}$, we retained that having symmetry closest to centrosymmetry. Both BP and TPamine moieties introduce torsional degrees of freedom leading to nonplanar optimal ground state geometries with twist angles of about $45^{\circ}$. Thus, the TPamine based chromophores adopt a propeller-shaped structure, the phenyl rings being twisted with respect to the trigonal planar nitrogen. The TPamine core allows for either left or right handed skewness of the propeller core but both geometries lead to comparable total energies. All these features are in line with those reported for related dipolar, ${ }^{8-10}$ quadrupolar ${ }^{16}$ and octupolar chromophores. ${ }^{8,9}$ Despite reduction of conjugation, as a result of the large twist angles, it has been demonstrate that significant electron communications is still in action and can lead to enhanced NLO responses. ${ }^{4}$

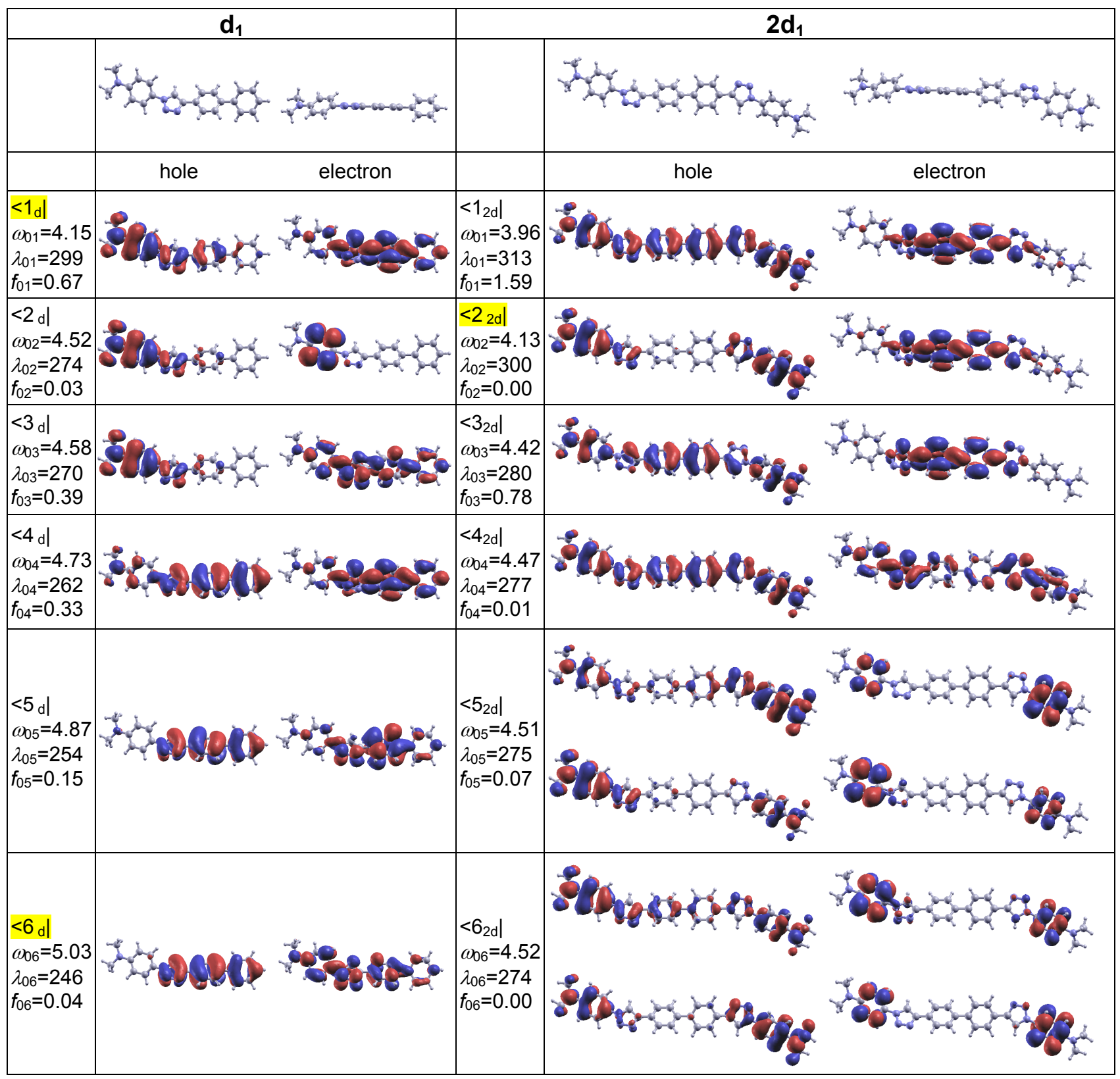

Figure 2. Optimized geometries of dipole $\mathbf{d}_{\mathbf{1}}$ and quadrupole $\mathbf{2} \mathbf{d}_{\mathbf{1}}$ and corresponding Natural Transition Orbitals ${ }^{33}$ (NTOs) of the first six excited states. Excited state numbers are given in the left panels, and additional values quote in sequence the associate transition energy $(\mathrm{eV})$, transition wavelength $(\mathrm{nm})$ and oscillator strength for OPA. Main TPA states are highlighted. 
The triazole moiety appears to introduce additional twist along the molecular backbone depending on the nature of the chromophore. Our findings are consistent with the structural parameters of related triazole based structures reported in the Cambridge Structural Data Base. ${ }^{39}$ For all investigated chromophores, the phenyl ring connected to the carbon atom of the triazole ring is systematically less twisted than that connected to the nitrogen atom. The former twist angle is almost vanishing in $\mathbf{d}_{1}$ and of about $10^{\circ}$ in $\mathbf{3 d}_{\mathbf{2}}$. That on the opposite side is almost the same for all four members of the series and of about $42^{\circ}$. This large twist angle is expected to significantly reduce electron communication towards the donating $\mathrm{NMe}_{2}$ groups and this effect will be discussed in the next sections thanks to NTO inspection.

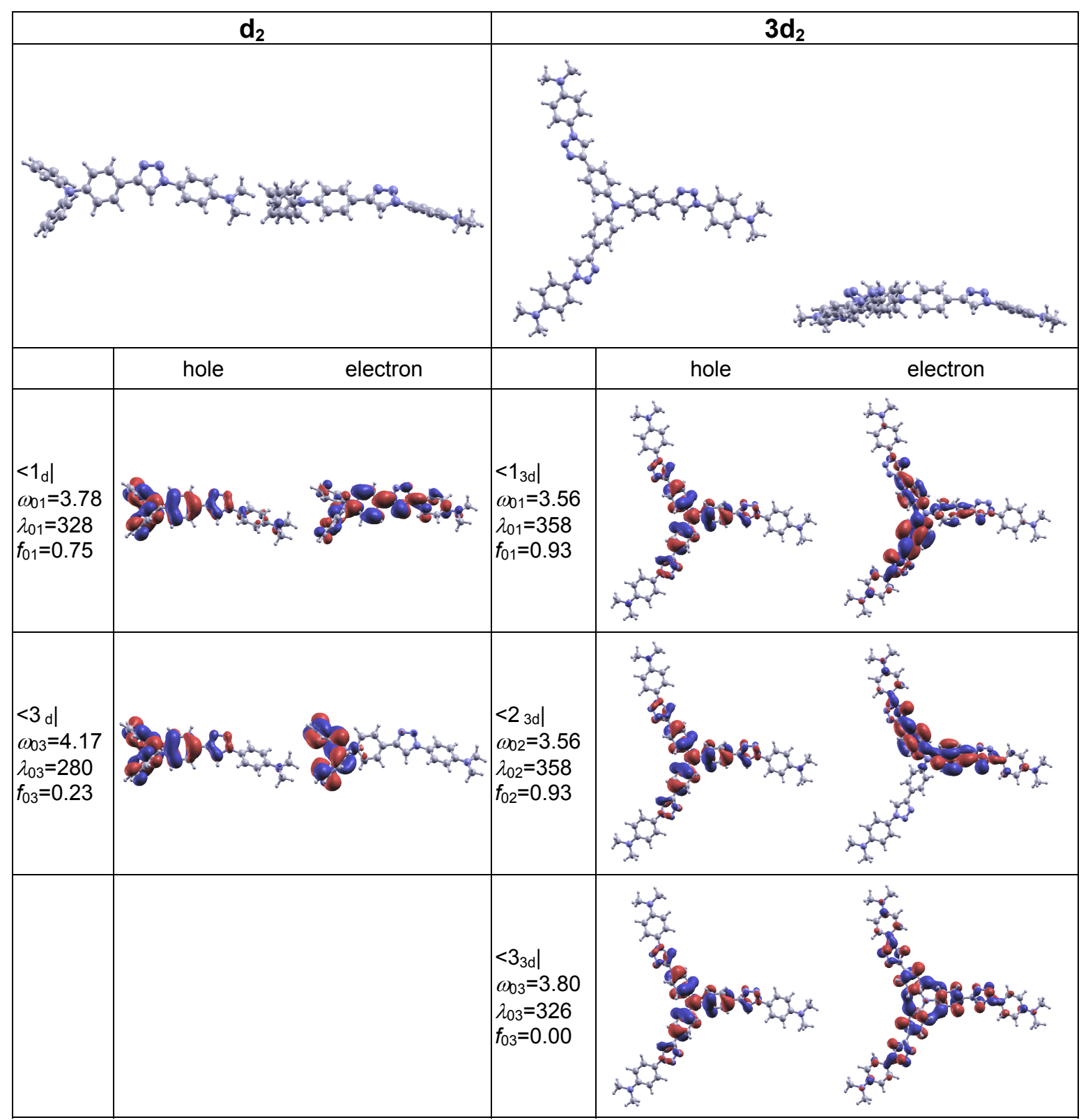




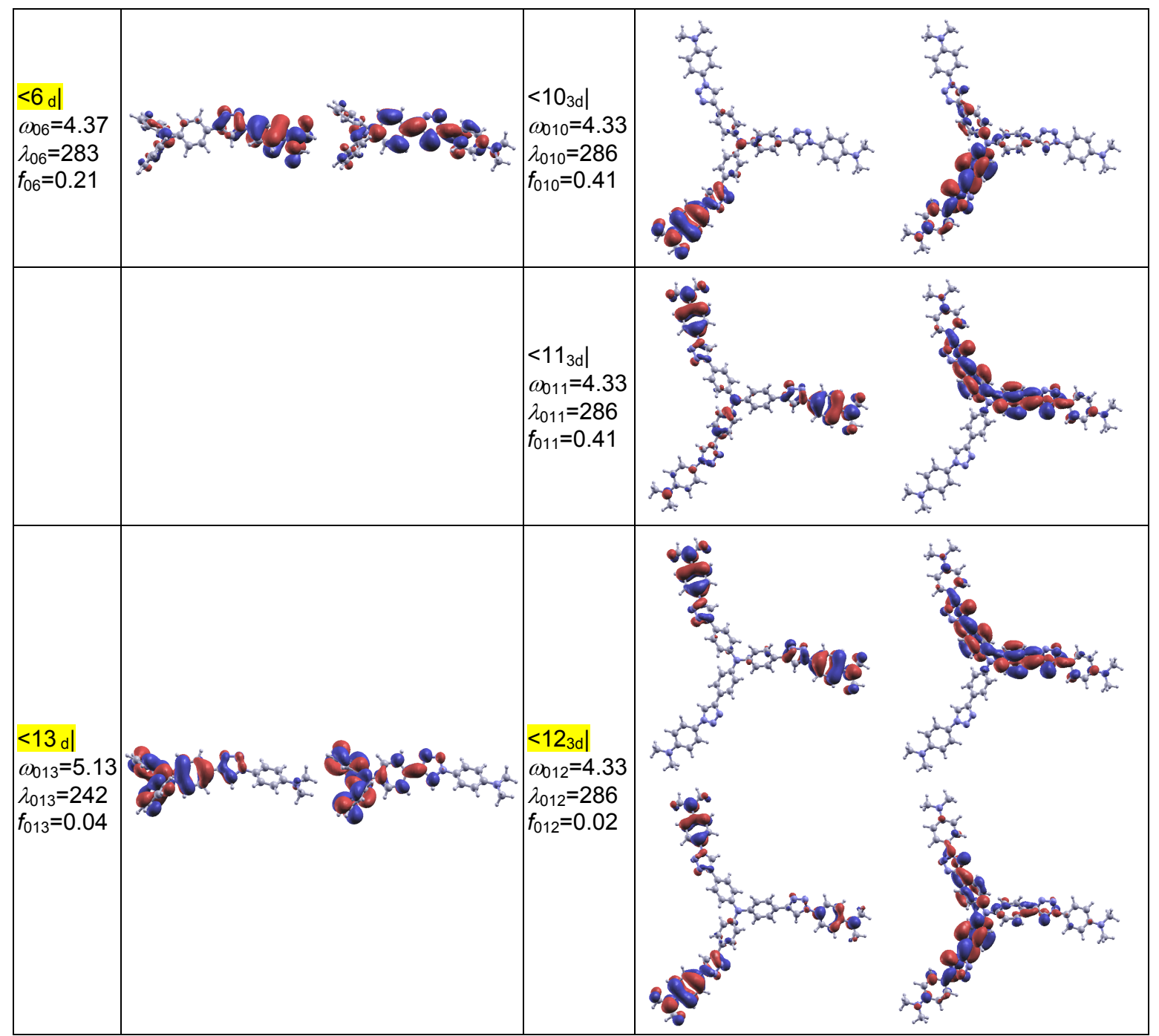

Figure 3. Optimized geometries of dipole $\mathbf{d}_{\mathbf{2}}$ and octupole $\mathbf{3} \mathbf{d}_{\mathbf{2}}$ and corresponding Natural Transition Orbitals ${ }^{33}$ (NTOs) of selected excited states. Excited state numbers are given in the left panels, and additional values quote in sequence the associate transition energy $(\mathrm{eV})$, transition wavelength $(\mathrm{nm})$ and oscillator strength for OPA. Main TPA states are highlighted.

\subsection{One Photon Absorption (OPA)}

First, let us validate the TD-DFT methodology used to determine the optical responses of the chromophores investigated in this work. Table 1 summarizes the available experimental photophysical data (in toluene) ) $^{31,32}$ and corresponding results obtained at the TD-B3LYP/6-31G level of theory for geometries optimized at the HF/6-31G level of theory, all in vacuum. The lowest transition energies (band gaps) show an overall good agreement, with shifts not larger than $0.15 \mathrm{eV}$. They bear all large oscillator strength, typical of such conjugated molecules. Assuming comparable linewidths for $\mathbf{2 d}_{1}$ and $\mathbf{3} \mathbf{d}_{2}$, the ratio between experimental molar extinction coefficients $(\varepsilon)$ associated to the first band can be compared to the ratio of calculated oscillator strength $\left(f_{01}^{\text {calc }}\right)$. The ratio of 1.17 obtained for $f_{01}^{\text {calc }}\left(\mathbf{3 d}_{\mathbf{2}}\right) / f_{01}^{\text {calc }}\left(\mathbf{2} \mathbf{d}_{\mathbf{1}}\right)$ compares nicely with the value of 1.20 found experimentally. Thus, we believe that the employed level of theory allows for accurate description of the excited states involved in the optical properties of interest and results are not expected to be plagued by difficulties such as spurious charge-transfer states. ${ }^{30}$ 
Table 1. Calculated ground state dipole moment $\mu_{00}$, experimental and calculated transition energies $\omega_{01}$ of the first excited state, corresponding calculated transition dipole moments $\mu_{01}$ and oscillator strengths $f_{01}$ normalized for the number of branches $N$, experimental molar extinction coefficients $\varepsilon$, experimental and calculated TPA cross sections $\sigma_{2}$ at $577 \mathrm{~nm}$.

\begin{tabular}{|l|l|l|l|l|l|l|l|l|c|}
\hline & $\begin{array}{c}\mu_{00}^{c a l c} \\
\mathbf{( D )}\end{array}$ & $\begin{array}{c}\omega_{01}^{\exp } \\
\mathbf{( e V )}\end{array}$ & $\begin{array}{c}\omega_{01}^{\exp } \\
(\mathbf{e V})\end{array}$ & $\begin{array}{c}\omega_{01}^{\text {calc }} \\
\mathbf{( e V )}\end{array}$ & $\begin{array}{c}\mu_{01}^{\text {calc }} \\
\mathbf{( D )}\end{array}$ & $f_{01}^{\text {calc }} / \mathbf{N}$ & $\mathbf{l o g} \mathbf{\varepsilon}$ & $\sigma_{2}^{\exp }$ & $\sigma_{2}^{\text {calc }}$ \\
\hline $\mathbf{d}_{\mathbf{1}}$ & 6.9 & $4.00^{a}$ & $4.00^{a}$ & 4.15 & 6.5 & 0.67 & - & - & 50 \\
\hline $\mathbf{2} \mathbf{d}_{\mathbf{1}}$ & 3.4 & $3.81^{a, b}$ & $3.81^{a, b}$ & 3.96 & 10.3 & 0.80 & $4.84^{a}$ & $99^{a}$ & 205 \\
\hline $\mathbf{d}_{\mathbf{2}}$ & 6.7 & - & - & 3.78 & 7.2 & 0.75 & - & - & 190 \\
\hline $\mathbf{3 d}_{\mathbf{2}}$ & 9.7 & $3.57^{a}$ & $3.57^{a}$ & 3.56 & $8.3^{c}$ & 0.62 & $4.92^{a}$ & $>240^{a}$ & 875 \\
\hline
\end{tabular}

${ }^{a}$ in toluene from ref. ${ }^{31}$

${ }^{b}$ in toluene from ref. ${ }^{32}$

${ }^{c}$ for each of the two degenerate excited states

Along the series $\mathbf{d}_{\mathbf{1}}, \mathbf{2} \mathbf{d}_{\mathbf{1}}, \mathbf{d}_{\mathbf{2}}, \mathbf{3} \mathbf{d}_{\mathbf{2}}$, the first excited state undergoes a systematic red shift while maintaining excellent transparency in the visible region. These findings corroborate experimental ones and confirm that these triazole based chromophores are good candidates for optical limiting in the visible region. ${ }^{31}$ Comparison to related dipolar, ${ }^{8-10}$ quadrupolar $^{40}$ and three-branched chromophores ${ }^{8,9,14,16,41}$ show a systematic blue shift of the first absorption band while maintaining good OPA cross sections. The latter are reduced by about $15 \%$ when compared to chromophores where the triazole ring is replaced by either a double or triple bond in the connecting unit between the branching center and the donating end group. ${ }^{10,40,41}$ This decrease is comparable to that induced by either replacing the $\mathrm{NHex}_{2}$ end group by an electron acceptor such as $\mathrm{SO}_{2} \mathrm{Oct}$ or $\mathrm{SO}_{2} \mathrm{CF}_{3}{ }^{10,40,41}$ or by introduction of a slight electronic dissymmetry ${ }^{37}$ and allows at the same time for larger blue shifts and thus improved transparency in the visible. For these triazole based chromophores, this reduction is directly related to the large twist angle between the triazole ring and the phenyl ring connected to the end-group. In fact, comparison of the NTOs corresponding to the first excitation (Figures 2 and 3 ) of each chromophore to those of related compounds (Figure 3 of ref. ${ }^{10}$ for $\mathbf{d}_{1}$; Figures S4-S5 of ref. ${ }^{16}$ for $\mathbf{2}_{1}$; Figure 7 of ref. ${ }^{8}$ for $\mathbf{d}_{\mathbf{2}}$ and $\mathbf{3}_{\mathbf{2}}$ ) shows reduced delocalization towards the donating end group best visible on corresponding electrons, thus decreasing the amount of charge transfer upon excitation. We underline that both hole and electron of $<1_{3 \mathrm{~d}} \mid$ and $<1_{\mathrm{d}}$ (Figure 3) essentially involve the central TPamine core as this will have interesting consequences on their TPA responses.
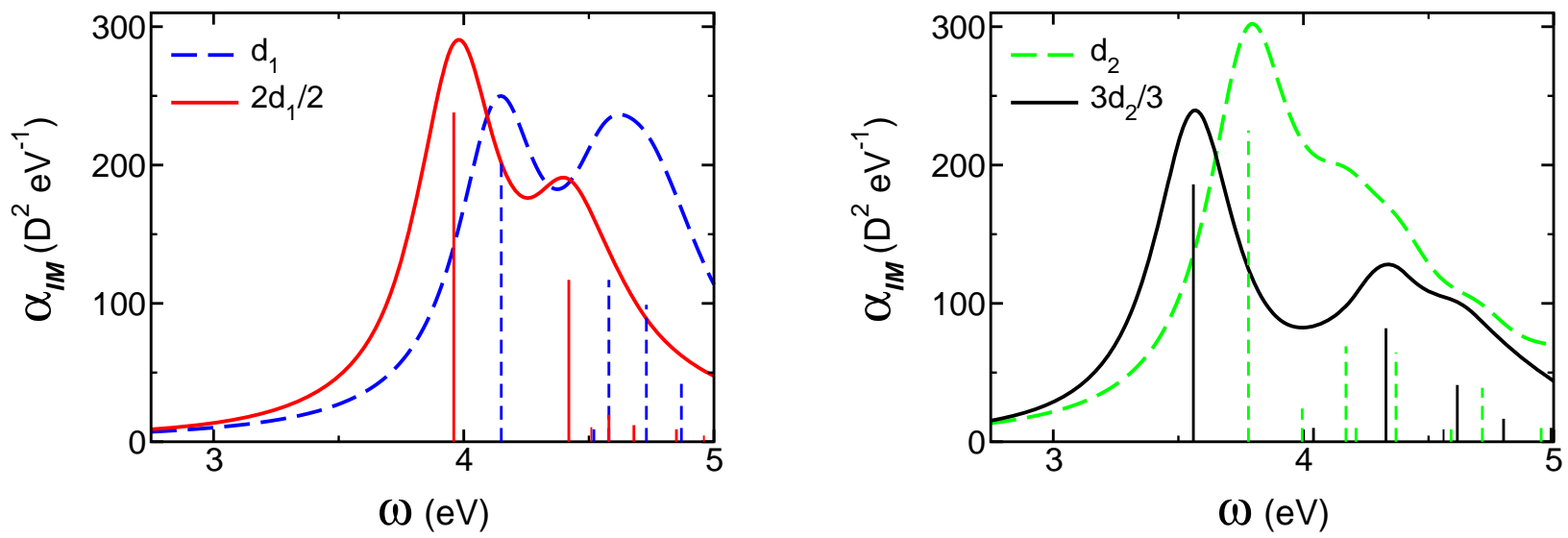

Figure 4. Calculated absorption spectra, normalized for the number of branches, of dipole $\mathbf{d}_{\mathbf{1}}$ and quadrupole $\mathbf{2} \mathbf{d}_{\mathbf{1}}$ (left panel) and dipole $\mathbf{d}_{\mathbf{2}}$ and octupole $\mathbf{3} \mathbf{d}_{\mathbf{2}}$ (right panel). Solid and dashed sticks represent relative oscillator strengths. 
In addition, normalized oscillator strength reported in Table 1 and absorption spectra shown in Figure 4 illustrate the different behavior of absorption upon branching. While the first absorption band is significantly enhanced when gathering two dipoles $\mathbf{d}_{\mathbf{1}}$ to obtain quadrupole $\mathbf{2} \mathbf{d}_{1}$, gathering three dipoles $\mathbf{d}_{\mathbf{2}}$ into $\mathbf{3} \mathbf{d}_{\mathbf{2}}$ decreases its amplitude. Again, NTOs are quite useful to understand these trends. In fact, the BP core allows for increased delocalization of the hole of $<1_{2 \mathrm{~d}} \mid$ as compared to that of $<1_{\mathrm{d}} \mid$ (Figure 2), while spreading over the triazole moiety is slightly reduced both for the hole and the electron associated to $<1_{3 \mathrm{~d}} \mid$ when compared to $<1_{\mathrm{d}}$ (Figure 3 ).

Finally, all compounds show additional close higher lying excited states bearing significant oscillator strength (Figures 2-4) which may show up in experiment as additional bands or band broadening depending on the amount of homogeneous and inhomogeneous broadening.

\subsection{Relevance of the Frenkel exciton scheme}

From the large red shift of the first absorption bands of $\mathbf{2} \mathbf{d}_{\mathbf{1}}$ and $\mathbf{3} \mathbf{d}_{\mathbf{2}}$ (Table 1 and Figure 4), the Frenkel exciton model (Figure 1) predicts significant interbranch couplings $V$ of 0.19 and $0.22 \mathrm{eV}$ respectively. However, inspection of transition energies of higher lying excited states is essential to validate this simple picture. In addition, NTOs are also quite useful to decide whether each dipole can be well approximated by a two-level system when viewed as the molecular building block corresponding to the branched structure. For the first two excited state of quadrupole $\mathbf{2 d}_{1}$, the exciton picture is nor fully relevant neither completely inappropriate. In fact, comparison of the NTOs of $<1_{d} \mid$ and $\left(<1_{2 d} \mid\right.$, $<2_{2 \mathrm{~d}}$ ) show the ability of the BP core to favour significant conjugation between both sides of the quadrupole (figure 2). This leads to the stabilization of the first four excited states. Each of these four states does not result from a single dipolar excited state but from a mixture of the lowest excited states having significant weight on the BP moiety either for the electron or both for the hole and the electron (e.g. $\left.<4_{d} \mid\right)$. Thus, the energies of the first two excited states of the quadrupole do not satisfy the splitting predicted by the exciton model (Figure 1). On the contrary, a very nice correspondence is observed between state $<2_{\mathrm{d}} \mid$ and states $\left(<5_{2 \mathrm{~d}} \mid,<6_{2 \mathrm{~d}}\right)$. The latter are multiconfigurational and can be represented only by a pair of NTOs. They correspond to bonding and anti-bonding combinations of monomeric state $<2_{\mathrm{d}}$ l leading to anti-symmetric (even parity) and symmetric (odd parity) transitions respectively. This is a direct consequence of excitation localization on the peripheral moieties as the gathering of the two dipolar unit in a twobranched quadrupolar structure will not allow significant interaction. Thus these two quadrupolar states have comparable transition energies (splitting of about $0.005 \mathrm{eV}$ ) and almost the same as state $<2_{\mathrm{d}} \mid$.

The situation is somewhat different for dipole $\mathbf{d}_{\mathbf{2}}$ and three-branched chromophore $\mathbf{3} \mathbf{d}_{\mathbf{2}}$. In fact, comparison of NTOs for state $<1_{\mathrm{d}} \mid$ and $\left(<1_{3 \mathrm{~d}}\left|,<2_{3 \mathrm{~d}}\right|,<3_{3 \mathrm{~d}} \mid\right)$ reveal good correspondence (Figure 3$)$. However, corresponding transition energies are far from the $(-V,+2 V)$ splitting predicted within the exciton model (Figure 1), while it was the case for related octupoles. ${ }^{4}$ Here, both hole and electron have large weight on the TPamine branching center allowing for significant through bond interbranch communication that stabilizes the first three excited states of $\mathbf{3 d}_{\mathbf{2}}$. While the same holds regarding NTOs of $<6_{\mathrm{d}} \mid$ versus $\left(<10_{3 \mathrm{~d}}\left|,<11_{3 \mathrm{~d}}\right|,<12_{3 \mathrm{~d}}\right)$, the three later states are almost degenerated and only slightly stabilized. This has to be related to the location of the hole on the peripheral donating moieties. It suggests no or little interbranch communication and no sizeable enhancement of any of the optical responses for these excited states.

\subsection{Fluorescence solvatochromism}

Even so this work is limited to ground state geometries relevant for absorption, it may be of some help to understand the different emission behaviors reported experimentally. ${ }^{31,32}$ In fact, it is well known that for such molecular structures, relaxation of the excited state leads to planarization of the molecular backbone and increased conjugation in the relaxed excited state. The torsional degree of freedom related to the BP core ${ }^{16}$ adds to those surrounding the triazole ring, leading to a larger Stokes shift ${ }^{31}$ for $\mathbf{2} \mathbf{d}_{\mathbf{1}}$ than for $\mathbf{3 d}_{\mathbf{2}}$, as the TPamine undergoes little changes upon relaxation. ${ }^{8,9}$ Furthermore, excitation localization on one of the molecular branches prior to emission has been predicted and experimentally observed both for quadrupoles ${ }^{16,20}$ and three-branched chromophores. ${ }^{8,9,16,21}$ As a consequence, emission is expected to stem from a dipolar state, well localized over a single branch, leading to significant solvatochromism of the fluorescence. The major solvatochromic behavior of $\mathbf{2} \mathbf{d}_{1}$ as compared to $\mathbf{3 d}_{\mathbf{2}}{ }^{32}$ may also be a direct consequence of BP planarization in the relaxed excited state, which should result in a larger increase of the excited state dipole moment $\mu_{11}^{\text {emission }}$, ground state dipole moments $\mu_{00}$ of $\mathbf{d}_{\mathbf{1}}$ and $\mathbf{d}_{\mathbf{2}}$ being comparable (Table1). 


\subsection{Two Photon Absorption (TPA)}

Calculated TPA spectra, normalized for the number of branches, are reported in Figure 5. The main features revealed by these spectra are coincident peak position of the first TPA band of each branched chromophore and its dipolar counterpart, significant enhancement of TPA cross section for branched chromophores in the low energy part of the spectra, and large responses at higher energies related to high lying excited states such as $<6_{d} \mid$ for $\mathbf{d}_{1}$ (Figure 2) and $<13_{d} \mid$ for $\mathbf{d}_{\mathbf{2}}$ (Figure 3). Interestingly, while the position of the first TPA band corresponds well to half the energy of states $<1_{d} \mid$ and $<2_{2 \mathrm{~d}}$ of $\mathbf{d}_{1}$ and $\mathbf{2} \mathbf{d}_{1}$ respectively, no TPA maximum shows up near half the energies of states $<1_{d} \mid$ or $<3_{3 d}$ of $\mathbf{d}_{\mathbf{2}}$ and $\mathbf{3 d}_{\mathbf{2}}$ respectively. This corroborates experimental findings ${ }^{31}$ inserted into Table 1 as the TPA maximum of $\mathbf{2} \mathbf{d}_{1}$ seems to have been reached close to $577 \mathrm{~nm}$, whereas TPA of $\mathbf{3 d}_{\mathbf{2}}$ still increases at $577 \mathrm{~nm}$. Unfortunately, further comparison between experimental results and calculations is difficult. First, published experimental data is limited to single wavelength data for $\mathbf{2} \mathbf{d}_{\mathbf{1}}$ and $\mathbf{3} \mathbf{d}_{\mathbf{2}}$. In addition, both experiments and calculations may suffer from inherent difficulties and are subject to differences regarding conventions used for the definition of underlying physical quantities. ${ }^{4}$ From the theoretical point of view, we recall that linewidths (scaling as $1 / \Gamma$ is expected for amplitudes in a simple few states model) are kept identical for all members of the series and no solvent effect has been accounted for. ${ }^{4}$
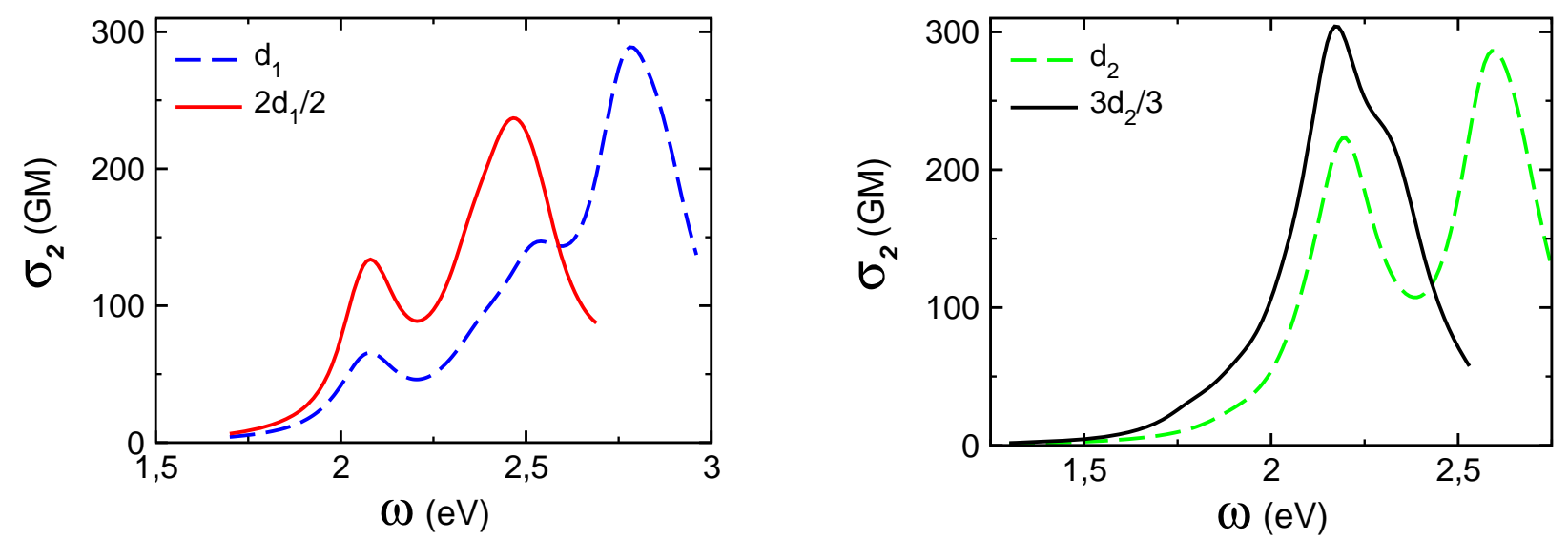

Figure 5. Calculated TPA cross section normalized for the number of branches of dipole $\mathbf{d}_{\mathbf{1}}$ and quadrupole $\mathbf{2} \mathbf{d}_{\mathbf{1}}$ (left panel) and dipole $\mathbf{d}_{\mathbf{2}}$ and octupole $\mathbf{3} \mathbf{d}_{\mathbf{2}}$ (right panel).

Reasons for concomitant positions of the first TPA band and TPA enhancement are quite different for $\mathbf{d}_{\mathbf{1}}$ and $\mathbf{d}_{\mathbf{2}}$ based chromophores. As already discussed in the previous section, excited state $<2_{2 d} \mid$ of $\mathbf{2} \mathbf{d}_{1}$ results from a mixture of different dipolar excited states. Compared to $<1_{\mathrm{d}} \mid$ of $\mathbf{d}_{1}$, both holes and electrons are more localized on the end groups and BP core respectively, thus increasing its charge transfer character. Combined to the increase of transition dipole moment $\mu_{01}$, related to significant through BP core communication, TPA is enhanced by a factor of two (Figure 5). As a result, the main TPA bands qualitatively agree with the exciton picture (Figure 1), but strong through bond interactions invalidate the model as a tool for quantitative analysis. The situation is, in some sense, even worse for chromophores $\mathbf{d}_{\mathbf{2}}$ and $\mathbf{3} \mathbf{d}_{\mathbf{2}}$. Indeed, while the main absorption band of $\mathbf{d}_{\mathbf{2}}$ corresponds to $<1_{\mathrm{d}}$, the first TPA band mainly arises from $<6_{\mathrm{d}}$ ( Figures 3 and 5). NTOs illustrate the corresponding electronic redistribution: the hole is no more localized on the central TPamine core but involves the donating end group with an extension up to the triazole moiety, while the electron extends over the whole spacer connecting the two nitrogen atoms. Thus, introduction of the triazole ring decouples OPA and TPA states, and induces a dramatic blue shift of the TPA spectrum. The TPA response of $\mathbf{3} \mathbf{d}_{\mathbf{2}}$ can be viewed alike, the main TPA band being related to $<12_{3 \mathrm{~d}} \mid$ which shows a marked correspondence with $<6_{\mathrm{d}}$ ( Figures 3 and 5). As no splitting between $\left(<10_{3 \mathrm{~d}}\left|,<11_{3 \mathrm{~d}}\right|\right)$ and $<12_{3 \mathrm{~d}}$ is observed, the enhancement of the TPA cross section (per branch, Figure 5) of $\mathbf{3}_{\mathbf{2}}$ with respect to $\mathbf{d}_{\mathbf{2}}$ has to be related to coherent interactions between branches (beyond the Frenkel exciton model where $<6_{\mathrm{d}} \mid$ would be the main monomeric excited state). Such enhancement has already been reported for other three-branched TPamine based chromophores (Figure 15 of ref. ${ }^{4}$ ). As no local-field, nor refractive index corrections (Figure 9 of ref. ${ }^{4}$ ) have been taken into account in the present work, the enhancement of $\mathbf{3 d}_{\mathbf{2}}$ versus $\mathbf{d}_{\mathbf{2}}$ is less important. This observation could be a direct consequence from hole localization on peripheral groups which may significantly reduce the efficiency of both through space and coherent interbranch interactions. 


\section{CONCLUSION}

Based on state of the art TD-DFT calculations, this work allows for a better understanding of the specific behavior of multipolar triazole-based chromophores. Such compounds can be synthesized thanks to "click" chemistry ${ }^{31}$ and have been shown to be promising candidates for optical limiting in the visible region. ${ }^{31,32}$

Overall decrease of OPA and TPA responses, when compared to related multipolar fluorophores, are directly related to significant conjugation disruption next to the triazole ring which leads to significant decrease of transition dipole moments. The specific effect upon branching is also quite different from analogous structures and different for the two branched systems investigated in this study. While quadrupole $\mathbf{2} \mathbf{d}_{\mathbf{1}}$ shows enhanced OPA and TPA responses when compared to twice those of its dipolar counterpart $\mathbf{d}_{\mathbf{1}}$, the three-branched chromophore $\mathbf{3}_{\mathbf{2}}$ reveals reduced OPA but enhanced TPA responses with respect to three times those of the molecular unit $\mathbf{d}_{\mathbf{2}}$. On the other hand, the first TPA maxima of both branched compounds occur almost at the same energy as that of their dipolar monomers.

First of all, the peripheral nitrogen end-groups play a quite different role in both series: it acts as a donating moiety in $\mathbf{d}_{1}$ and $\mathbf{2} \mathbf{d}_{1}$, while it does almost not participate in the first few excited states of $\mathbf{d}_{\mathbf{2}}$ and $\mathbf{3} \mathbf{d}_{\mathbf{2}}$. This is a direct consequence of possible electron-withdrawing character of BP core ${ }^{10,16}$ as compared to the electron donating one of the TPAmine core. In addition, the BP core of $\mathbf{2} \mathbf{d}_{\mathbf{1}}$ allows for significant mixing of the dipolar excited states while the TPamine core retains a good correspondence between excited states of $\mathbf{d}_{\mathbf{2}}$ and $\mathbf{2} \mathbf{d}_{1}$, well illustrated thanks to NTOs. Thus, the through bond communication resulting from the BP core significantly stabilizes the first excited states of $\mathbf{2} \mathbf{d}_{\mathbf{1}}$ and increases both OPA and TPA oscillator strengths. On the other hand, in $\mathbf{d}_{\mathbf{2}}$ and $\mathbf{3}_{\mathbf{2}}$ the triazole moiety triggers the competition between the two nitrogen-based donating end groups which contribute either to the first OPA or first TPA band. These bands are related to different excited states both in the dipole (first versus sixth excited state) and in the three-branched system (first two versus twelfth excited state). It results in a dramatic blue shift of the position of the first TPA band with respect to twice the OPA transition energy. Additionally, TPA cross section of the first band is significantly enhancement with respect to that of quadrupole $\mathbf{2} \mathbf{d}_{\mathbf{1}}$. We underline that such behavior is unusual as quadrupoles have commonly better normalized (either for the number of branches, the molecular weight or the number of effective electrons ${ }^{42}$ ) TPA cross sections than related three-branched chromophores. ${ }^{4}$

Finally, the present study provides interesting perspectives for TPA-transparency and fluorescence probes optimization. Indeed, the better understanding of the subtle-acting of the triazole moiety may be combined to complementary routes for molecular engineering. Fluorene core instead of BP core should allow for increased conjugation along the quadrupolar backbone and both improved OPA and TPA responses. ${ }^{16,40}$ Replacement of donating end-groups by electron-withdrawing moities should lead to further blue shifts and major changes of the role played by the triazole ring. ${ }^{8,9,40}$ Variation of the triazole substituent positioning may induce significant structural modifications and dramatic changes of both linear and NLO responses. ${ }^{43}$ Slight dissymmetrical functionalization could also offer interesting perspectives for spatial and spectral control of the fluorescence properties while maintaining good TPA cross sections. ${ }^{37}$

\section{ACKNOWLEDGMENTS}

This work was performed in part at the US Department of Energy, Center for Integrated Nanotechnologies (CINT), at Los Alamos National Laboratory (LANL) (contract DE-AC52-06NA25396). This work was granted access to the HPC resources of CINES under the allocation 2005-[c20050822414] and 2008-[x20080825087] made by GENCI (Grand Equipement National de Calcul Intensif).

\section{REFERENCES}

[1] Kim, H. M. and Cho, B. R., "Two-photon materials with large two-photon cross sections. Structure-property relationship," Chemical Communications (2), 153-164 (2009).

[2] Pawlicki, M., Collins, H. A., Denning, R. G. and Anderson, H. L., "Two-Photon Absorption and the Design of TwoPhoton Dyes," Angewandte Chemie-International Edition 48(18), 3244-3266 (2009).

[3] He, G. S., Tan, L. S., Zheng, Q. and Prasad, P. N., "Multiphoton absorbing materials: Molecular designs, characterizations, and applications," Chemical Reviews 108(4), 1245-1330 (2008). 
[4] Terenziani, F., Katan, C., Badaeva, E., Tretiak, S. and Blanchard-Desce, M., "Enhanced Two-Photon Absorption of Organic Chromophores: Theoretical and Experimental Assessments," Advanced Materials 20(24), 4641-4678 (2008).

[5] Joshi, M. P., Swiatkiewicz, J., Xu, F. M. and Prasad, P. N., "Energy transfer coupling of two-photon absorption and reverse saturable absorption for enhanced optical power limiting," Optics Letters 23(22), 1742-1744 (1998).

[6] Chung, S. J., Kim, K. S., Lin, T. H., He, G. S., Swiatkiewicz, J. and Prasad, P. N., "Cooperative enhancement of twophoton absorption in multi-branched structures," Journal of Physical Chemistry B 103(49), 10741-10745 (1999).

[7] Cho, B. R., Son, K. H., Lee, S. H., Song, Y. S., Lee, Y. K., Jeon, S. J., Choi, J. H., Lee, H. and Cho, M. H., "Two photon absorption properties of 1,3,5-tricyano-2,4,6-tris(styryl)benzene derivatives," Journal of the American Chemical Society 123(41), 10039-10045 (2001).

[8] Katan, C., Terenziani, F., Mongin, O., Werts, M. H. V., Porres, L., Pons, T., Mertz, J., Tretiak, S. and BlanchardDesce, M., "Effects of (multi)branching of dipolar chromophores on photophysical properties and two-photon absorption," Journal of Physical Chemistry A 109(13), 3024-3037 (2005).

[9] Katan, C., Terenziani, F., Droumaguet, C. L., Mongin, O., Werts, M. H. V., Tretiak, S. and Blanchard-Desce, M., "Branching of dipolar chromophores: effects on linear and nonlinear optical properties," Proc. SPIE 5935, 593503 (2005).

[10] F. Terenziani, C. Le Droumaguet, C. Katan, Mongin, O. and Blanchard-Desce, M., "Effect of branching on twophoton absorption in triphenylbenzene derivatives," Chemphyschem 8(5), 723-734 (2007).

[11] Bartholomew, G. P., Rumi, M., Pond, S. J. K., Perry, J. W., Tretiak, S. and Bazan, G. C., "Two-photon absorption in three-dimensional chromophores based on 2.2 -paracyclophane," Journal of the American Chemical Society 126(37), 11529-11542 (2004).

[12] Drobizhev, M., Karotki, A., Dzenis, Y., Rebane, A., Suo, Z. Y. and Spangler, C. W., "Strong cooperative enhancement of two-photon absorption in dendrimers," Journal of Physical Chemistry B 107(31), 7540-7543 (2003).

[13] Yoo, J., Yang, S. K., Jeong, M. Y., Ahn, H. C., Jeon, S. J. and Cho, B. R., "Bis-1,4(p-diarylaminostryl)-2,5dicyanobenzene derivatives with large two-photon absorption cross-sections," Organic Letters 5(5), 645-648 (2003).

[14] Mongin, O., Porres, L., Katan, C., Pons, T., Mertz, J. and Blanchard-Desce, M., "Synthesis and two-photon absorption of highly soluble three-branched fluorenylene-vinylene derivatives," Tetrahedron Letters 44(44), 8121-8125 (2003).

[15] Varnavski, O., Yan, X. Z., Mongin, O., Blanchard-Desce, M. and Goodson, T., "Strongly interacting organic conjugated dendrimers with enhanced two-photon absorption," Journal of Physical Chemistry C 111(1), 149-162 (2007).

[16] Katan, C., Tretiak, S., Werts, M. H. V., Bain, A. J., Marsh, R. J., Leonczek, N., Nicolaou, N., Badaeva, E., Mongin, O. and Blanchard-Desce, M., "Two-photon transitions in quadrupolar and branched chromophores: Experiment and theory," Journal of Physical Chemistry B 111(32), 9468-9483 (2007).

[17] Lambert, C., Schmalzlin, E., Meerholz, K. and Brauchle, C., "Synthesis and nonlinear optical properties of threedimensional phosphonium ion chromophores," Chemistry-a European Journal 4(3), 512-521 (1998).

[18] Lambert, C., Noll, G., Schmalzlin, E., Meerholz, K. and Brauchle, C., "Synthesis, (non)linear optical and redox properties of a donor-substituted truxenone derivative," Chemistry-a European Journal 4(11), 2129-2135 (1998).

[19] Beljonne, D., Wenseleers, W., Zojer, E., Shuai, Z. G., Vogel, H., Pond, S. J. K., Perry, J., W., Marder, S., R. and Bredas, J., L., "Role of dimensionality on the two-photon absorption response of conjugated molecules: The case of octupolar compounds," Advanced Functional Materials 12(9), 631-641 (2002).

[20] Terenziani, F., Painelli, A., Katan, C., Charlot, M. and Blanchard-Desce, M., "Charge instability in quadrupolar chromophores: Symmetry breaking and solvatochromism," Journal of the American Chemical Society 128(49), 1574215755 (2006).

[21] Terenziani, F., Sissa, C. and Painelli, A., "Symmetry breaking in octupolar chromophores: Solvatochromism and electroabsorption," Journal of Physical Chemistry B 112(16), 5079-5087 (2008).

[22] Champagne, B., Guillaume, M. and Zutterman, F., "TDDFT investigation of the optical properties of cyanine dyes," Chemical Physics Letters 425(1-3), 105-109 (2006).

[24] Tretiak, S. and Chernyak, V., "Resonant nonlinear polarizabilities in the time-dependent density functional theory," Journal of Chemical Physics 119(17), 8809-8823 (2003).

[25] Masunov, A. M., Tretiak, "Prediction of two-photon absorption properties for organic chromophores using timedependent density-functional theory," Journal of Physical Chemistry B 108(3), 899-907 (2004).

[26] Grimme, S., "Calculation of the electronic spectra of large molecules," Reviews in Computational Chemistry 20, 153-218 (2004).

[27] Dreuw, A. and Head-Gordon, M., "Single-reference ab initio methods for the calculation of excited states of large molecules," Chemical Reviews 105(11), 4009-4037 (2005). 
[28] Casida, M. E., Jamorski, C., Casida, K. C. and Salahub, D. R., "Molecular excitation energies to high-lying bound states from time-dependent density-functional response theory: Characterization and correction of the time-dependent local density approximation ionization threshold," Journal of Chemical Physics 108(11), 4439-4449 (1998).

[29] Furche, F. and Ahlrichs, R., "Adiabatic time-dependent density functional methods for excited state properties," Journal of Chemical Physics 117(16), 7433-7447 (2002).

[30] Magyar, R. J. and Tretiak, S., "Dependence of spurious charge-transfer excited states on orbital exchange in TDDFT: Large molecules and clusters," Journal of Chemical Theory and Computation 3(3), 976-987 (2007).

[31] Parent, M., Mongin, O., Kamada, K., Katan, C. and Blanchard-Desce, M., "New chromophores from click chemistry for two-photon absorption and tuneable photoluminescence," Chemical Communications (15), 2029-2031 (2005).

[32] Zoon, P. D., Stokkum, I. H. M. v., Parent, M., Mongin, O., Blanchard-Desce, M. and Brouwer, A. M., "Fast photoprocesses in triazole-based push-pull systems," Physical Chemistry Chemical Physics 12(11), 2706-2715 (2010).

[33] Martin, R. L., "Natural transition orbitals," Journal of Chemical Physics 118(11), 4775-4777 (2003).

[34] Frisch, M. J., Trucks, G. W., Schlegel, H. B., Scuseria, G. E., Robb, M. A., Cheeseman, J. R., Montgomery Jr., J. A., Stratmann, R. E., Burant, J. C., Dapprich, S., Millam, J. M., Daniels, A. D., Kudin, K. N., Strain, M. C., Farkas, O., Tomasi, J., Barone, V., Cossi, M., Cammi, R., Mennucci, B., Pomelli, C., Adamo, C., Clifford, S., Ochterski, J., Petersson, G. A., Ayala, P. Y., Cui, Q., Morokuma, K., Malick, D. K., Rabuck, A. D., Raghavachari, K., Foresman, J. B., Cioslowski, J., Ortiz, J. V., Stefanov, B. B., Liu, G., Liashenko, A., Piskorz, P., Komaromi, I., Gomperts, R., Martin, R. L., Fox, D. J., Keith, T., Al-Laham, M. A., Peng, C. Y., Nanayakkara, A., Gonzalez, C., Challacombe, M., Gill, P. M. W., Johnson, B., Chen, W., Wong, M. W., Andres, J. L., Head-Gordon, M., S., R. E. and Pople, J. A., Gaussian 98, Revision A.11, Gaussian, Inc., Pittsburgh PA (2001).

[35] Frisch, M. J., Trucks, G. W., Schlegel, H. B., Scuseria, G. E., Robb, M. A., Cheeseman, J. R., Montgomery, Jr., J. A., Vreven, T., Kudin, K. N., Burant, J. C., Millam, J. M., Iyengar, S. S., Tomasi, J., Barone, V., Mennucci, B., Cossi, M., Scalmani, G., Rega, N., Petersson, G. A., Nakatsuji, H., Hada, M., Ehara, M., Toyota, K., Fukuda, R., Hasegawa, J., Ishida, M., Nakajima, T., Honda, Y., Kitao, O., Nakai, H., Klene, M., Li, X., Knox, J. E., Hratchian, H. P., Cross, J. B., Bakken, V., Adamo, C., Jaramillo, J., Gomperts, R., Stratmann, R. E., Yazyev, O., Austin, A. J., Cammi, R., Pomelli, C., Ochterski, J. W., Ayala, P. Y., Morokuma, K., Voth, G. A., Salvador, P., Dannenberg, J. J., Zakrzewski, V. G., Dapprich, S., Daniels, A. D., Strain, M. C., Farkas, O., Malick, D. K., Rabuck, A. D., Raghavachari, K., Foresman, J. B., Ortiz, J. V., Cui, Q., Baboul, A. G., Clifford, S., Cioslowski, J., Stefanov, B. B., Liu, G., Liashenko, A., Piskorz, P., Komaromi, I., Martin, R. L., Fox, D. J., Keith, T., Al-Laham, M. A., Peng, C. Y., Nanayakkara, A., Challacombe, M., Gill, P. M. W., Johnson, B., Chen, W., Wong, M. W., Gonzalez, C. and Pople, J. A., Gaussian 03, Revision D.02, Gaussian, Inc.: Wallingford CT (2004).

[36] Onida, G., Reining, L., Rubio, A., "Electronic excitations: density-functional versus many-body Green's-function approaches," Review of Modern Physics 74(2), 601-659 (2002).

[37] Katan, C., Charlot, M., Mongin, O., Le Droumaguet, C., Jouikov, V., Terenziani, F., Badaeva, E., Tretiak, S. and Blanchard-Desce, M., "Simultaneous Control of Emission Localization and Two-Photon Absorption Efficiency in Dissymmetrical Chromophores," The Journal of Physical Chemistry B 114(9), 3152-3169 (2010).

[38] Kokalj, A., "Computer graphics and graphical user interfaces as tools in simulations of matter at the atomic scale," Computational Materials Science 28(2), 155-168 (2003).

[39] Allen, F. H., "The Cambridge Structural Database: a quarter of a million crystal structures and rising," Acta Crystallographica 58(1), 380-388 (2002).

[40] Mongin, O., Porres, L., Charlot, M., Katan, C. and Blanchard-Desce, M., "Synthesis, fluorescence, and two-photon absorption of a series of elongated rodlike and banana-shaped quadrupolar fluorophores: A comprehensive study of structure-property relationships," Chemistry-a European Journal 13(5), 1481-1498 (2007).

[41] Porres, L., Mongin, O., Katan, C., Charlot, M., Pons, T., Mertz, J. and Blanchard-Desce, M., "Enhanced two-photon absorption with novel octupolar propeller-shaped fluorophores derived from triphenylamine," Organic Letters 6(1), 4750 (2004).

[42] Kuzyk, M. G., "Fundamental limits on two-photon absorption cross sections," Journal of Chemical Physics 119(16), 8327-8334 (2003).

[43] Jarowski, P. D., Wu, Y. L., Schweizer, W. B., Diederich , F., "1,2,3-triazoles as conjugative pi-linkers in push-pull chromophores: Importance of substituent positioning on intramolecular charge-transfer," Organic Letters 10(15), 33473350 (2008).

*claudine.katan@insa-rennes.fr; phone +33 682580335; fax +33 223238618; http://www.umr6510.univ-rennes1.fr/ and

http://foton.insa-rennes.fr/efoton-insa.html 